Background The COVID-19 pandemic persisted for most of 2020 and, although the government initially canceled thousands of events, the need for adaptation forced many of them to go online. Through various digital platforms, people found new ways to deal with social distance and, at the same time, to promote events.

Methods A two-step approach was used for this analysis: 1) creation of a data collection form using Google Forms, and 2) transfer of data from the form to a spreadsheet using Google Spreadsheets.

Results The Ministry of Health of Brazil (MoH Brazil) started hosting webinars and virtual meetings. Between July and December 2020, MoH Brazil held four thematic webinars focusing on the Young Key Populations (YKP). As a result, more than 600 YKP were trained to deal with social vulnerabilities, especially with mental health and behavioral issues.

Conclusion The use of this post-event databank will help governments to identify ways to move forward with the presentation of information and to organize trainings based on YKP interests. These webinars also represent a virtual safe space that engage the youth in a healthy discussion regarding social protection measures that are adapted to the covid-19 context.

\section{P424 RAPIDLY RISING EPIDEMIC OF AFRICAN PLASMID/ BLATEM-1 IN PENICILLINASE-PRODUCING NEISSERIA GONORRHOEAE FROM GUANGDONG, CHINA}

X Qin, H Zheng. Dermatology Hospital, Southern Medical University, Guangzhou, China

10.1136/sextrans-2021-sti.446

Background Our previous study showed a recent increase in the incidence of African-type penicillinase-producing Neisseria gonorrhoeae (PPNG) in Guangdong, China. The aim of this study was to investigate the prevalence and molecular epidemiology of African plasmid in PPNG isolates in Guangdong in two time frames, 2013-2015 and 2018-2019.

Methods A total of 864 isolates were collected in two cities in Guangdong. Minimum inhibitory concentrations (MICs) of seven antimicrobials were determined by the agar dilution method. The molecular epidemiological characteristics were determined by PPNG and tetracycline-resistant $\mathrm{N}$. gonorrhoeae (TRNG) plasmids typing, Sanger sequencing of TEM $\beta$-lactamase (blaTEM) genes,NG_porB gene and N. gonorrhoeae multiantigen sequence typing (NG-MAST).

Results Investigation by PCR showed that 31.02\% (268/864) of the isolates were PPNG, of which 53.36\% (143/268) contained the TRNG plasmid. The blaTEM genes of PPNG isolates were carried by African increased (18.42\% to $66.67 \%$ ), Asian decreased $(81.58 \%$ to $28.89 \%)$, and Toronto/Rio increased (0 to 4\%) plasmids. Further plasmid typing showed that PPNG isolates consisted of three major clusters, namely African plasmid/blaTEM-1 (128/268, 47.76\%), Asian plasmid/ blaTEM-135?(59/268, 22.01\%), and Asian plasmid/blaTEM-1 $(57 / 268,21.27 \%)$. The percentage of isolates with the blaTEM-1-carrying African plasmid as predominant plasmid increased significantly from $13.16 \%(5 / 38)$ in 2013 to $66.67 \%$ (60/90) in 2019. Among the isolates carrying the African plasmid possessing blaTEM-1, NG-MAST_sequence type (ST) 5061, ST1927, ST17748 and NG_porB type 8, 13,11,12 were found to be the predominant STs in both periods, respectively, but which showed differences in two cities in two periods.
Conclusions The proportion of African plasmid/blaTEM-1 PPNG has continuously increased since 2013, in association with clonal spread, which might contribute to the rising gonorrhoea epidemic in Guangdong. The possibility that African plasmid/blaTEM-1 was acquired requires careful follow-up and continuous monitoring of African plasmid/blaTEM-1 to ascertain whether it constitutes a step towards evolutionary change.

\section{P425 PREVALENCE OF NEISSERIA GONORRHOEAE IN INDIA: A SYSTEMATIC LITERATURE REVIEW}

${ }^{1} \mathrm{P}$ Thomas*, ${ }^{1} \mathrm{R}$ Kant, ${ }^{1 \mathrm{~J}}$ Lal, ${ }^{2} \mathrm{~S}$ Morre, ${ }^{2} \mathrm{P}$ Thomas. ${ }^{1}$ Sam Higginbottom University of Agriculture, Technology and Science, Prayagraj, India; ${ }^{2}$ Institute of Public Health genomics, Maastricht, The Netherlands

\subsection{6/sextrans-2021-sti.447}

Background This paper provides an update the current state of knowledge on the status of C. trachomatis (CT) in India. CT is one of the most common Sexually Transmitted Infections (STIs) in the world, yet evidence on the burden in developing countries, as India is scarce.

Results This paper includes all the studies containing C. trachomatis testing that were identified in the literature search for the literature review on gonorrhea in India. The papers were included from PubMed, EMBASE and Google Scholar. The studies that contained data on CT were considered for further inclusion. Studies were included in this review if they contained C. trachomatis testing data

Results This paper provides 31 new studies that were not featured in the previous review. Prevalence of CT in the included studies ranges from $0.4 \%$ to $29 \%$. The studies are classified in four patient categories namely: OPD and STI clinic attendees, Commercial sex workers and their clients, Men who have sex with men and Trans genders and population groups. The most commonly reported testing method was PCR. This paper helps to provide a broader picture regarding C. trachomatis in India by providing prevalence levels for some groups of high relevance for STIs, like Commercial Sex Workers (CSW) and Men who have Sex with Men (MSM). This review provides more ample background in the CT burden in India among the most vulnerable and fringe groups. This paper also raises the issue of referencing of research papers on STIs in India to facilitate review and research on the matter

\section{P426 BURDEN OF C. TRACHOMATIS IN INDIA, AN UPDATE ON EVIDENCE FROM THE LITERATURE}

'J Narayan Yada*, ${ }^{1} \mathrm{R}$ Kant, ${ }^{1} \mathrm{~J}$ Lal, ${ }^{2} \mathrm{~S}$ Morre, ${ }^{2} \mathrm{P}$ Thomas. ${ }^{1}$ Sam Higginbottom University of Agriculture, Technology and Sciences, Prayagraj, India; ${ }^{2}$ Institute of Public Health genomics, Maastricht University, Maastricht, The Netherlands

\subsection{6/sextrans-2021-sti.448}

Background This paper provides an update the current state of knowledge on the status of C. trachomatis (CT) in India. CT is one of the most common Sexually Transmitted Infections (STIs) in the world, yet evidence on the burden in developing countries, as India is scarce.

Results This paper includes all the studies containing C. trachomatis testing that were identified in the literature search for the literature review on gonorrhea in India. The papers were included from PubMed, EMBASE and Google Scholar. The studies that contained data on CT were considered for 
further inclusion. Studies were included in this review if they contained C. trachomatis testing data

Results This paper provides 31 new studies that were not featured in the previous review. Prevalence of CT in the included studies ranges from $0.4 \%$ to $29 \%$. The studies are classified in four patient categories namely: OPD and STI clinic attendees, Commercial sex workers and their clients, Men who have sex with men and Trans genders and population groups. The most commonly reported testing method was PCR. This paper helps to provide a broader picture regarding C. trachomatis in India by providing prevalence levels for some groups of high relevance for STIs, like Commercial Sex Workers (CSW) and Men who have Sex with Men (MSM). This review provides more ample background in the CT burden in India among the most vulnerable and fringe groups. This paper also raises the issue of referencing of research papers on STIs in India to facilitate review and research on the matter

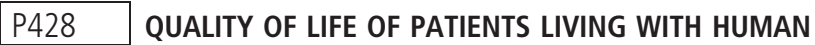 IMMUNODEFICIENCY VIRUS INFECTION - EVIDENCE FROM SOUTH INDIA}

S Katpattil * . Kerala University of Health Sciences, India

10.1136/sextrans-2021-sti.449

Introduction With anti-retroviral therapy (ART) for human immunodeficiency virus infection (HIV) coming into picture, quality of life (QOL) has gained importance. Knowledge on the factors affecting QOL would be helpful in making important policy decisions and health care interventions. The aim of this study is to assess the quality of life of people living with HIV (PLWH) and to identify the factors influencing their QOL.

Materials and Methods The study was done among 100 PLWH attending a tertiary care hospital, and three Non-Governmental Organizations atCalicut, Kerala, India, from June 2015 to May 2018. QOL was assessed using HIV specific World Health Organization Quality Of Life scale (WHOQOL-HIV) - BREF questionnaire which has six domains (physical, psychological, level of independence, social relationships, environment and spirituality/religiousness/personal belief). Social support and stigma were measured using 'Multidimensional Scale of Perceived Social Support' and 'HIV Stigma Scale,' respectively, using Likert Scale. Factors influencing QOL were identified using backward stepwise multiple linear regression with the six domain scores as the dependent variables.

Results Male:Female ratio was $1: 1$ and 58\% were in early stage of the disease (stage I/II). Psychological and SRPB (Spirituality Religiousness and Personal Beliefs) domains were the most affected domains. All the regression models were statistically significant $(\mathrm{P}<0.05)$. The determination coefficient was highest for the social relationship domain (57\%) followed by the psychological domain (51\%). Disease stage and perceived social support significantly influenced all the domains of WHOQOL. Younger age, female gender, rural background, shorter duration of HIV, non-intake of ART and greater HIV related stigma were the high risk factors of poor QOL.

Conclusion Interventions such as ART, family, vocational and peer counselling would address these modifiable factors influencing QOL, thereby improving the QOL of PLWH.

\section{P429} HEALTH RELATED QUALITY OF LIFE AMONG HIV AFFECTED INDIVIDUALS - A CROSS SECTIONAL STUDY

A Sha*. Eids, India

\subsection{6/sextrans-2021-sti.450}

Introduction Identifying the level of health related quality of life (HQoL) and their influencing factors in human immunodeficiency virus (HIV) positive people is of extreme importance in implementing an interventional program to support this group. This cross sectional study was an attempt to determine the level and factors associated with HQoL among the individuals affected with HIV.

Methodology A convenient sample of 82 HIV-infected people from three NGOs and one Infectious Disease Hospital (IDH), were interviewed using an interviewer administered, semi structured questionnaire developed by adopting the 'WHOQOL-HIV BREF instrument'.

Results Majority of the respondents were with low Quality of Life (QoL) in all the domains of HQoL. The proportion of respondents with low QoL was highest in the domain of social relationship (64.6\%) followed by psychological domain (59.8\%), physical domain (58.5\%), level of independence domain (56.1\%), environmental domain (52.4\%) and spirituality domain $(52.4 \%)$ of HQoL. Bivariate analysis revealed that the overall perception of QoL was better in the respondents living in urban area, who were employed and asymptomatic of the centre for disease control (CDC) stage of HIV.

Conclusion The perception of overall health was higher in females, all respondents less than 35 years of age, asymptomatic of the CDC stage of disease and with a current CD4 count greater than $200 \mathrm{cell} / \mathrm{mm} 3$. These findings highlight the need for enhanced socio-psychosocial support and a better environment for improving the health related quality of life among individuals affected with HIV.

\section{P430 A PEOPLE-CENTERED APPROACH TO DEVELOP INTERVENTION PACKAGES FOR HIV PARTNER NOTIFICATION: FACILITATORS AND BARRIERS UNDER A SOCIOECOLOGICAL FRAMEWORK}

X Yan*, Y Xu, W Tang, J Tucker, W Miller. UNC Project-China

\subsection{6/sextrans-2021-sti.451}

Background The rate of HIV infections among MSM in China has been on the rise in recent years despite public health efforts to reach key populations for HIV prevention. The limited acceptability and usage of partner notification services (PS) would be one reason. People-centered approach such as crowdsourcing, which collects ideas from the public to solve a certain problem, may be promising for developing more effective intervention packages in promoting PS.

Methods This study used mixed methods to develop PS intervention strategies and analyze emerging themes of facilitators and barriers for PS. First, we used a community-based participatory approach to organize a crowdsourcing contest that solicited innovative works for promoting PS among MSM in China. Second, descriptive analysis was used to examine the demographic characteristics of the participants and the features of the eligible entries. Finally, we conducted content analysis using inductive and deductive coding methods under 